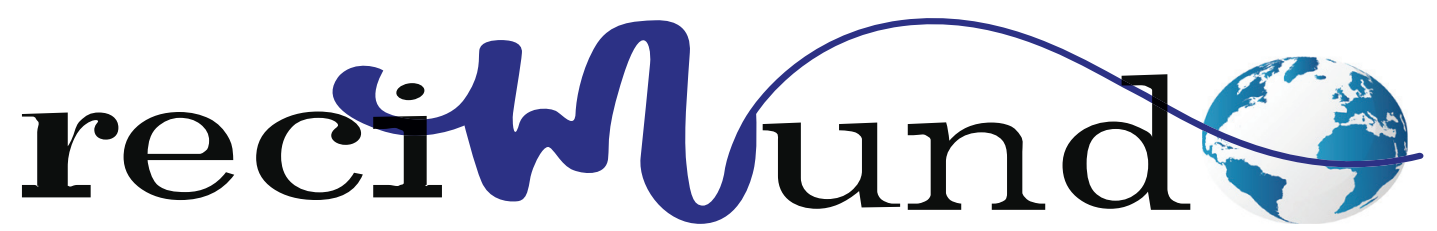

Revista Científica Mundo de la Investigación y el Conocimiento

DOI: $10.26820 /$ recimundo/4.(1).esp.marzo.2020.164-172

URL: http://recimundo.com/index.php/es/article/view/779

EDITORIAL: Saberes del Conocimiento

REVISTA: RECIMUNDO

ISSN: 2588-073X

TIPO DE INVESTIGACIÓN: Artículo de Revisión

CÓDIGO UNESCO: 5801 Teoría y Métodos Educativos

PAGINAS: $164-172$

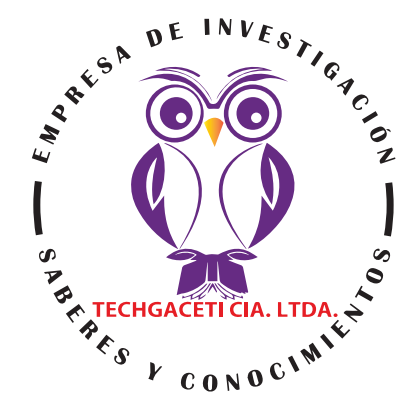

\title{
Aprendizaje humanista y su aplicabilidad en aula de clases
}

\section{Humanistic learning and its applicability in the classroom}

\section{Aprendizagem humanística e sua aplicabilidade na sala de aula}

\begin{abstract}
Douglas Vicente Vásquez Aguilar'; Luis Enrique Soto Chávez²; Arturo Enrique Sanchez Granja3;
\end{abstract}
Lidia Baldramira Chang Camacho ${ }^{4}$

RECIBIDO: 20/11/2019 ACEPTADO: 29/01/2020 PUBLICADO: 05/03/2020

1. Magister en Diseño Curricular; Diploma Superior en Pedagogía Universitaria; Licenciado en Ciencias Sociales y Polóticas; Abogado de los Tribunales y Juzgados de la República del Ecuador; Docente Facultad Ingeniería Industrial; Universidad de Guayaquil; Guayaquil, Ecuador; douglas.vasqueza@hotmail.com; (DD https://orcid. org/0000-0003-2403-6916

2. Magister Universitario en Ingenieria Avanzada de Producción Logística y Cadena de Suministro; Docente Facultad Ingeniería Industrial; Universidad de Guayaquil; Guayaquil, Ecuador; josel.mosquerav@ug.edu.ec; (DD https://orcid.org/0000-0001-8854-6881

3. Magister en Ciencias Ambientales; Ingeniero Industria; Docente Facultad Ingeniería Industrial; Universidad de Guayaquil; Guayaquil, Ecuador; arturo.sanchezg@ug.edu.ec; (D) https://orcid.org/0000-0003-2449-2428

4. Magister en Seguridad, Higiene Industrial y Salud Ocupacional; Magister en Psicología Laboral con Mención en Desarrollo Humano y de Organización; Ingeniera Industrial; Docente Facultad Ingeniería Industrial; Universidad de Guayaquil; Guayaquil, Ecuador; lidia.changca@ug.edu.ec; (D) https://orcid.org/0000-0002-6308-0832

CORRESPONDENCIA

Douglas Vicente Vásquez Aguilar

douglas.vasqueza@hotmail.com

\section{Guayaquil, Ecuador}




\section{RESUMEN}

Este trabajo de investigación academica denominado aprendizaje humanista científico, deberiá ser el rol permanente del docente en la facultad Ingeniería Industrial Universidad de Guayaquil en este nuevo siglo, esperamos que sea puesto en práctica por los compañeros Docentes en las diferentes Universidades públicas y privadas del Ecuador, desde la perspectiva académica y con toda la experiencia obtenida por el docente en el desarrollo cognitivo permanente en el aula de clases se construye el conocimiento entre el profesor y el estudiante, con el paradigma aprendizaje humanista en el que el eje central es el estudiante y el docente facilitador deberá otorgarle todas las herramientas didácticas pedagógicas para el aprendiente pueda fortalecer sus conocimientos académicos y continuar con su proceso de estudios superiores, con el objetivo formar excelentes profesionales humanistas técnicos en el área de la Ingeniería Industrial, desde la vigencia de la Constitución de la República del Ecuador hecho acontecido el 20 de Octubre del año 2008, en Ciudad Alfaro Montecristi Manabí, la Educación superior inicio un nuevo proceso de cambio, paradigma diferente, en la Universidad de Guayaquil, inicio el proceso de formación y superación académica para que el claustro Docente obtenga títulos de posgrados en las diferentes líneas de investigación el propósito fortalecer conocimiento del docente, y consecuentemente optimizar el desempeño de los estudiantes los mismos que deberán ser competitivos, innovadores, humanistas, para que aporten al desarrollo sostenible y sustentable del Ecuador, además, en la Facultad de Ingeniería Industrial de la Universidad de Guayaquil, en la actualidad ya se imparte el proceso académico de enseñanza aprendizaje con Humanismo, valores, conocimiento integral, por competencias, cumpliendo con el mandato de la Constitución de la República del Ecuador misma que determina lo siguiente, Art. 26. - La educación es un derecho de las personas a lo largo de su vida y un deber ineludible e inexcusable del Estado. Constituye un área prioritaria de la política pública y de la inversión estatal, garantía de la igualdad e inclusión social y condición indispensable para el buen vivir. Las personas, las familias y la sociedad tienen el derecho y la responsabilidad de participar en el proceso educativo. Art. 27.- La educación se centrará en el ser humano y garantizará su desarrollo holístico, en el marco del respeto a los derechos humanos, al medio ambiente sustentable y a la democracia; será participativa, obligatoria, intercultural, democrática, incluyente y diversa, de calidad y calidez; impulsará la equidad de género, la justicia, la solidaridad y la paz; estimulará el sentido crítico, el arte y la cultura física, la iniciativa individual y comunitaria, y el desarrollo de competencias y capacidades para crear y trabajar. La educación es indispensable para el conocimiento, el ejercicio de los derechos y la construcción de un país.

Palabras clave: Aprendizaje, Humanista, Holístico, Axiológico, Epistemológico.

\section{ABSTRACT}

This academic research work called scientific humanist learning, should be the permanent role of the teacher in the Industrial Engineering faculty University of Guayaquil in this new century, we hope it will be put into practice by fellow Teachers in the different public and private universities of Ecuador, From the academic perspective and with all the experience obtained by the teacher in the permanent cognitive development in the classroom, knowledge is built between the teacher and the student, with the humanistic learning paradigm in which the central axis is the student and the The facilitator teacher must grant all the pedagogical didactic tools for the learner to strengthen their academic knowledge and continue with their process of higher studies, with the aim of training excellent technical humanistic professionals in the area of Industrial Engineering, from the validity of the Constitution of I To the Republic of Ecuador, which occurred on October 20, 2008, in Ciudad Alfaro Montecristi Manabí, Higher Education began a new process of change, a different paradigm, at the University of Guayaquil, began the process of training and academic improvement so that the Teaching staff obtain postgraduate degrees in the different lines of research in order to strengthen teacher knowledge, and consequently optimize student performance, which must be competitive, innovative, humanistic, to contribute to the sustainable and sustainable development of Ecuador, in addition, at the Faculty of Industrial Engineering of the University of Guayaquil, the academic teaching-learning process with Humanism, values, integral knowledge, by competences is already being taught, complying with the mandate of the Constitution of the Republic of Ecuador, same which determines the following. Art. 26.- Education is a right of people throughout their lives and an inescapable and inexcusable duty of the State. It constitutes a priority area of public policy and state investment, guarantee of social equality and inclusion and an indispensable condition for good living. Individuals, families and society have the right and responsibility to participate in the educational process. Art. 27.- Education will focus on the human being and will guarantee its holistic development, within the framework of respect for human rights, the sustainable environment and democracy; It will be participatory, compulsory, intercultural, democratic, inclusive and diverse, of quality and warmth; will promote gender equity, justice, solidarity and peace; It will stimulate critical sense, art and physical culture, individual and community initiative, and the development of skills and abilities to create and work. Education is indispensable for knowledge, the exercise of rights and the construction of a country.

Keywords: Learning, Humanist, Holistic, Axiological, Epistemological.

\section{RESUMO}

Este trabalho de pesquisa acadêmica, chamado aprendizado humanista científico, deve ser o papel permanente do professor da Universidade de Guayaquil, na faculdade de Engenharia Industrial, neste novo século, esperamos que seja colocado em prática por colegas professores nas diferentes universidades públicas e privadas do Equador. , Do ponto de vista acadêmico e com toda a experiência obtida pelo professor no desenvolvimento cognitivo permanente em sala de aula, o conhecimento é construído entre o professor e o aluno, com o paradigma humanístico de aprendizagem em que o eixo central é o aluno e o facilitador. o professor deve conceder todas as ferramentas didáticas pedagógicas para que o aluno fortaleça seus conhecimentos acadêmicos e continue seu processo de estudos superiores, com o objetivo de formar excelentes profissionais humanistas técnicos na área de Engenharia Industrial, a partir da validade da Constituição de República do Equador, ocorrida em 20 de outubro de 2008, em Ciudad Alfaro Montecristi Manabí, Ensino Superior iniciou um novo processo de mudança, um paradigma diferente, na Universidade de Guayaquil, iniciou o processo de treinamento e aprimoramento acadêmico para que os docentes obtenham pós-graduação nas diferentes linhas de pesquisa, a fim de fortalecer o conhecimento dos professores. e, consequentemente, otimizar o desempenho do aluno, que deve ser competitivo, inovador, humanístico, para contribuir para o desenvolvimento sustentável e sustentável do Equador. Além disso, na Faculdade de Engenharia Industrial da Universidade de Guayaquil, o processo de ensino-aprendizagem acadêmico com humanismo valores, conhecimento integral, por competências, já está sendo ensinado, cumprindo o mandato da Constituição da República do Equador, mesmo que determina o seguinte: art. 26.- A educação é um direito das pessoas durante toda a vida e um dever inescapável e indesculpável do Estado. Constitui uma área prioritária de políticas públicas e investimentos do Estado, garantia de igualdade e inclusão social e condição indispensável para o bem-estar. Indivíduos, famílias e sociedade têm o direito e a responsabilidade de participar do processo educacional. Arte. 27.- A educação se concentrará no ser humano e garantirá seu desenvolvimento holístico, no âmbito do respeito aos direitos humanos, ao meio ambiente sustentável e à democracia; Será participativo, obrigatório, intercultural, democrático, inclusivo e diversificado, de qualidade e cordialidade; promoverá a equidade de gênero, justiça, solidariedade e paz; Estimulará o senso crítico, a arte e a cultura física, a iniciativa individual e comunitária e o desenvolvimento de habilidades e habilidades para criar e trabalhar. A educação é indispensável para o conhecimento, o exercício dos direitos e a construção de um país.

Palavras-chave: Aprendizagem, Humanística, Holística, Axiológica, Epistemológica. 


\section{Introducción}

En Ecuador la educación superior publica en este nuevo siglo deberá ser eminentemente humanista, desde la perspectiva académica y con toda la experiencia obtenida por el docente en el desarrollo cognitivo permanente en el aula de clases se construye el conocimiento entre el profesor y el estudiante, con el paradigma aprendizaje humanista en el que el eje central será siempre el estudiante y el docente un facilitador o guía que deberá otorgarle todas las herramientas didácticas pedagógicas para el aprendiente pueda fortalecer sus conocimientos académicos y continuar con su proceso de estudios superiores, con el objetivo formar excelentes profesionales humanistas técnicos en el área de la Ingeniería Industrial, es un reto muy importante que deben desempeñar docentes de la Facultad de Ingeniería Industrial Universidad de Guayaquil, con formación continua en la parte axiología, epistemológica, holística, desarrollando en el aula de clases la praxis, humanismo, constituyendo avance académico en la investigación, con propuestas de innovación permanente en Autogogos y Hodogogo, basada en competencias educativas, la misma permitirá obtener mejores egresados con excelente información profesional en el campo laboral buscando la optimización del desarrollo de competencias de acuerdo al perfil de la carrera de Ingeniería Industrial en el transcurso del tiempo los aprendientes han observado que los contenidos de las asignaturas se encuentran muy desactualizados y no responden a las actuales expectativas que plantea la propia sociedad porque afecta seriamente el desempeño académico del estudiante por el desconocimiento de términos y conceptos que son de utilidad en la vida práctica, los futuros profesionales de la Facultad de Ingeniería Industrial de la Universidad de Guayaquil deberán tener formación Humanista.

\section{Desarrollo}

\section{Aporte cognitivo:}

Rol del docente humanista en el sector publico y privado del ecuador

\section{Nuevo Paradigma humanista}

Consideramos que desde nuestra representación como docentes de la Facultad Ingeniería Industrial Universidad de Guayaquil y por nuestra propia experiencia en el dictado de clases por varios años consecutivos hemos observado que es necesario implementar un nuevo paradigma educativo denominado Aprendizaje Humanista mismo que optimizara el conocimiento de los estudiantes de la carrera Ingeniería Industrial, seguros estamos que por los resultados obtenidos en los aprendientes mediante trabajos exposiciones colectivas e individuales en primer, segundo parcial, será el paradigma humanista el más importante en el desarrollo académico de los estudiantes por que permite la retro alimentación del conocimiento en la educación superior, aprende el estudiante y consecuentemente el profesor, en nuestras actividades diarias de Docentes descubrimos que en el aula de clases con talleres o trabajos académicos prácticos interactuados se construye el conocimiento, por varias ocasiones nos tocó interactuar con brillantes estudiantes observando un excelente desarrollo cognitivo, los profesores también aprende de ellos, el proceso de enseñanza aprendizaje debe ser bilateral que permita descubrir, extraer el conocimiento del estudiante para ayudarlo sus fortalezas y debilidades a cumplir su objetivo ser competitivo, buscando siempre la excelencia académica, para brindar un aporte muy significativo en el desarrollo sostenible y sustentable y humanista en el Ecuador, el Docente de la universidad de Guayaquil en este siglo debe centrarse de manera directa en el estudiante fortaleciendo permanentemente el aprendizaje, en la praxis el desarrollo integral del 
conocimiento, con valores éticos y morales, impulsando continuamente la Innovación, basada en competencias, utilizando herramientas informáticas actualizadas que permitan desde nuestras cátedras y por intermedio de teleconferencias de forma directa observando clases en línea en diferentes universidades nacionales y extranjeras y básicamente de países de habla hispana, de esta manera fomentaremos la praxis en el aula de clases, utilizando la metodología humanista se aprende haciendo o practicando en talleres a desarrollarse en aula de clases, desechando el antiguo paradigma conductista teórico en el que eje central Hera el profesor dueño absoluto de la verdad que jamás permitía el razonamiento del estudiante, el paradigma humanista constructivista en este nuevo siglo es el que permitirá al estudiante construir su propio conocimiento, el profesor se convierte en un guía o facilitador humanista del proceso de aprendizaje.

\section{Fundamentos teoricos}

TENDENCIAS EDUCATIVAS HUMANISTAS PARA EL SIGLO XXI EDUCACION VIRTUAL, ELEMENOS PARA LA DISCUSION (Por. Guillermo Cardona Ossa, Magíster en Educación U. Javeriana, Candidato a PhD Ciencias Pedagógicas) (tendencias educativas para el siglo XXI, 2008)

Según Los hombres son hombres, antes que médicos, ingenieros o arquitectos. La educación deberá hacer de ellos hombres honestos sensatos y capaces, y ellos con el tiempo se transformarán en médicos, ingenieros y arquitectos, honestos sensatos y capaces. (Jhon Stewart Miller,)

Hoy, educar es gobernar. Hace 150 años lo decía Domingo Faustino Sarmiento, el presidente que prefirió su humilde título de maestro "gobernar es educar". Así era ya en el siglo XIX. Así fue durante el siglo XX y así será, sobre todo, a medida que avance el nuevo siglo1.
Este va a ser el siglo del saber. Más precisamente el siglo de la racionalidad científica y tecnológica. Cierto: nuestra especie ha dependido siempre de sus creencias y sus tecnologías, desde el tiempo inmemorial del animismo y la invención de la rueda. Pero ahora esas creencias van siendo cada vez más penetradas por la ciencia y esas tecnologías están cambiando a un ritmo sin precedentes.

El saber cambia el mundo, y nuestro mundo está cambiando con la prontitud de los saberes nuevos. Por eso apenas atinamos a decir que nuestra época es distinta: hablamos de "posmodernidad" en la cultura y de post guerra fría en la geopolítica. Es como otro bigbang. El tiempo ahora es más corto, el espacio es más pequeño: lo uno se denomina "aceleración de la historia", lo otro es la "aldea global".

En la sociedad del conocimiento, la ciencia y la tecnología van conquistando los distintos ámbitos que comprenden la vida. Transformara nuestro modo de pensar, de sentir, y de actuar como aspectos fundamentales de lo cognitivo, lo axiológico y lo motor, dimensiones esenciales del hombre.

En segundo lugar, los oficios de la sociedad del conocimiento tienen un creciente contenido técnico y cada vez es mayor el número de ocupaciones de alta tecnología. No da lo mismo manejar un arado que manejar un tractor, una máquina de escribir que un computador, un bisturí que un rayo láser. Y en la cima de la pirámide ocupacional se encuentra una nueva clase de talentos, dedicados a diseñar soluciones únicas para problemas únicos. Cada día las competencias que exige la sociedad son más sofisticadas ya no es suficiente hablar de una profesión como Ingeniero o Administrador, sino que se exigirán unas competencias que cambiarán su perfil como, Ingeniero Administrativo de sistemas, Administrador de política de productos. 
Las industrias dinámicas de la sociedad del conocimiento son las industrias de la inteligencia: la biotecnología, la informática, la microelectrónica, las telecomunicaciones, la robótica, la industria de nuevos materiales y la aviación civil, entre otras son las actividades bandera del nuevo siglo. Dentro de un enfoque ecológico, ninguna de éstas GOMEZ BUENDIA, Educación La agenda del siglo XXI. Hacia un desarrollo humano. Industrias depende de los recursos naturales, ni de la mano de obra barata, ni siquiera del capital, que va a donde lo llaman con la velocidad de la luz. Estas industrias dependen de un nuevo factor de producción: se llama el conocimiento. En la sociedad del conocimiento el valor agregado ya no proviene de los factores clásicos de producción "tierra, capital y trabajo": viene de la tecnología antes que todo. Por ello la educación debe replantear sus objetivos, sus metas, sus pedagogías y sus didácticas si quiere cumplir con su misión en el siglo XXI, brindar satisfactores a las necesidades del hombre, como dice Bill Gates en lo que trae el futuro" Las mismas fuerzas tecnológicas que harán tan necesario el aprendizaje, lo harán agradable y practico. Las corporaciones se están reinventando en torno de las oportunidades abiertas por la tecnología de la información, las escuelas también tendrán que hacerlo. (Educacion agenda siglo XXI hacia un desarrollo Humano, 2010)

Parafraseando a nuestro Nobel Gabriel García Marqués "Creemos que las condiciones están dadas como nunca para el cambio social y que la educación será su órgano maestro. Una educación desde la cuna hasta la tumba, inconforme y reflexiva, que nos inspire un nuevo modo de pensar, quiénes somos en una sociedad que se quiere a sí misma. Que canalice hacia la vida la inmensa energía creadora que durante siglos hemos despilfarrado en la depredación y la violencia, y nos abra la segunda oportunidad sobre la tierra que no tuvo la estirpe desgraciada del coronel Aureliano Buendía. Por el país próspero que soñamos al alcance de los niños" (la educacion sera organo maestro desde la cuna hasta la tumba, 2008).

Navegar en la aldea global será una tarea que exige muy altos niveles de "inteligencia social" esto es, capacidad organizada del país para adaptarse a un mundo que cambia rápidamente, lo cual supone adquirir y procesar la información sumamente compleja, para tomar decisiones que aseguren el aprovechamiento óptimo de cada coyuntura. La alta inteligencia conlleva una ética de aceptación de la verdad, de responsabilidad, de autonomía, de compromiso con el bien público por encima de los intereses individuales o sectoriales, de respeto por los valores universales encarnados en los derechos humanos y en la práctica de la justicia para contribuir al logro de la paz. Por eso es necesario desarrollar el pensamiento crítico y estimular la actitud científica desde la primera escuela y a lo largo de toda su vida educativa.

La educación busca dentro de sus objetivos últimos la formación integral del ser humano, entendido como un ser de necesidades, habilidades y potencialidades. Busca intervenir en las Dimensiones Cognitivas (conocimientos) Axiológica (valores) y Motora (Habilidades y Destrezas), para mejorar la calidad de vida.

La virtualidad del saber no supone un saber-menos, o un saber-peor. Tampoco supone un saber-de-segundo grado o de segunda categoría. Antes bien, corresponde a una transformación de los procesos mediante los que se aprende, constata (anota) y explica el mundo, procesos que están en sintonía y dependencia con nivel de desarrollo de los medios tecnológicos del actual momento histórico. Por decirlo de otra manera: no existe garantía de que las setecientas páginas de la Crítica de la Razón Pura de Kant, conduzcan a mayor nivel de comprensión de la estructura del conocimiento (y, al cabo, a mejorar la felicidad del género 
humano) de las investigaciones neurológicas sobre el área del lenguaje, o los foros de discusión de Internet sobre el conocimiento intuitivo o el pensamiento creativo.

La virtualidad del conocimiento no estriba tanto en la metodología que guía a éste cuanto en los fenómenos a que da lugar. La Teoría de la Relatividad no posee mayor cualidad epistemológica por el hecho de que, como dijera Ortega y Gasset, en el momento en que fue formulada, apenas diez o doce personas en Europa se hallaban en condiciones de entenderla. Posiblemente, su importancia deriva de que ha conducido a una explicación del universo que, a juicio de la comunidad científica, supera, integrándolas, las cosmologías ptolemaica y newtoniana, y, además, ha llevado al hombre a la luna (y a los japoneses a perecer bajo un par de bombas atómicas). Pero la comunidad no-científica nada ha podido argüir al respecto. De la misma manera, el conocimiento dependiente de y circulante por las redes virtuales ( $y$, por tanto, sometido a sus constreñimientos') no posee menor ni mayor cualidad epistemológica por la forma en que se produce, ni por las leyes a que se sujeta. Como en el caso de las teorías einstenianas, es capaz de permitir diagnosis de (tendencias educativas para el siglo XXI, 2008)

\section{Educacion Humanista Siglo XXI}

La formación y la evaluación docente del profesorado universitario ante el espacio europeo de educación superior Juana María Madrid Izquierdo. (la formacion y evaluacion docente universitario en educacion superior europeo, 2005)

La creación del Espacio Europeo de Educación Superior necesita que el profesorado universitario tenga una formación docente adecuada. Los responsables administrativos y académicos de la universidad tienen que planificar y financiar esa formación. También, es necesario que la universidad valore e incentive la buena docencia (de la misma manera que ya lo viene haciendo con los méritos investigadores del profesorado). Para ello es necesaria una evaluación de la formación y una evaluación docente del profesorado. (la formacion y evaluacion docente universitario en educacion superior europeo, 2005)

Alma Elena Figueroa, María Gilio Medina, Victoria Gutiérrez Marfileño Expresan que es inminente el cambio en el campo de la educación, en general y en la formación profesional de los estudiantes universitarios, en particular. Desde la década de los sesenta y hasta la primera del siglo XXI, los planteamientos teóricos acerca del papel de la educación se modificaron y avanzaron a un ritmo más acelerado que las prácticas de enseñanza y aprendizaje, que requieren más tiempo para implantarse. En este artículo, se presenta el panorama de algunos de los principales organismos nacionales e internacionales que realizan, promueven, captan y/o sistematizan dichos cambios y que impactan la tendencia de la formación basada en competencias. Por otro lado, se presenta una lista de competencias docentes que el profesor necesita desarrollar para desempeñar su función con la mejor calidad posible; en especial, se muestran algunas relacionadas con su participación como ejecutor del currículum. Existe una discusión acerca del papel que juega la educación en general, y la formación de profesionistas de las instituciones de educación superior (IES) en particular, en los nuevos escenarios mundiales. La educación superior es un factor estratégico del desarrollo económico y tecnológico, capaz de resolver o anticiparse a problemas e identificar escenarios emergentes (Banco Mundial, 1995; Delgado, 2007), pero también debe estar alerta para convertirse en un factor clave del desarrollo humano y social (Delors, 1996)

La combinación de ambas preocupaciones generó lo que Tünnerman y Souza (2003) llaman la revolución copernicana en los 
procesos de enseñanza-aprendizaje, en la que se crean nuevas formas de conocer, de producir y de difundir conocimiento. Esto implica un cambio en el rol del docente, que debe orientar a los estudiantes a aprender a aprender y a hacerlo a través de distintos tipos de experiencias de aprendizaje dentro y fuera del aula, ligadas al ámbito laboral, en una época caracterizada por la abundancia de información que además se transforma a más velocidad que en décadas anteriores. La principal interrogante es ¿qué necesita hacer el docente para orientar el aprendizaje de los estudiantes bajo esta nueva perspectiva? El profesor pasará de ser un agente ocupado en exponer contenidos, a ser protagonista, un agente que participará y orientará de manera más activa el aprendizaje de sus estudiantes, más allá del aula en la modalidad presencial. Este cambio provocará que el profesor adquiera un rol de guía que analiza y orienta a los estudiantes en la solución de problemas o en la elaboración de proyectos reales y útiles. Planeará, ejecutará y evaluará adecuadamente experiencias sistemáticas de aprendizaje, más ligadas al mundo laboral; la docencia dejará de limitarse a proporcionar información e instrucciones aisladas y poco significativas para que los estudiantes aprendan; su principal función será la de guía que ofrezca organizada y pertinentemente rutas formativas de aprendizaje a sus estudiantes para que su aprendizaje implique un proceso formativo integral y de tal calidad, que pueda ser comparable, compatible y competitivo con cualquier otra institución de educación superior (la funcion del docente en la universidad, 2008)

\section{Métodos}

\section{Método Inductivo}

Se trata del método científico más usual, en el que pueden distinguirse cuatro pasos esenciales: la observación de los hechos para su registro; la clasificación y el estudio de estos hechos; la derivación inductiva que parte de los hechos y permite llegar a una generalización; y la contrastación.

\section{Método Deductivo}

El método deductivo es un método científico que considera que la conclusión se halla implícita dentro las premisas. Esto quiere decir que las conclusiones son una consecuencia necesaria de las premisas: cuando las premisas resultan verdaderas y el razonamiento deductivo tiene validez, no hay forma de que la conclusión no sea verdadera.

\section{Método analítico}

El Método analítico es aquel método de investigación que consiste en la desmembración de un todo, descomponiéndolo en sus partes o elementos para observar las causas, la naturaleza y los efectos

\section{Resultados}

Con la implementación del nuevo modelo educativo denominado Aprendizaje Humanista los resultados son muy positivos y halagadores en el desarrollo del conocimiento de los estudiantes de diferentes asignaturas en la Facultad de Ingeniería Industrial de la Universidad de Guayaquil Ecuador, se evidencian con las exposiciones colectivas e individuales y trabajos desarrollados en aula de clases por los estudiantes en primer y segundo parcial.

\section{Discusión}

Con el debate del tema aprendizaje humanista y permanente en el aula de clases el mismo que se discutió con varios Docentes y aproximadamente ciento diez estudiantes de la Facultad de Ingeniería Industrial de la Universidad de Guayaquil, se tomó la decisión de aportar e implantar este modelo educativo denominado Aprendizaje Humanista mismo que ayudaría al fortalecimiento académico de los estudiantes en la educa- 
ción superior Ecuatoriana.

\section{Conclusión}

En la universidad de Guayaquil y en cada una de sus unidades académicas de manera muy particular en la Facultad de Ingeniería Industrial, la educación superior en la actualidad impartida por sus profesores es de calidad, calidez, valores, epistemológica, humanista, excelente. Permite con absoluta libertad y confianza al estudiante demostrar sus conocimientos, construidos en el aula de clases, su propio desarrollo académico por intermedio del método aprendizaje humanista, como una herramienta diaria de trabajo en los espacios de aprendizaje, para los Docentes de Educación Superior en la Universidad de Guayaquil.

\section{Recomendación}

La Universidad de Guayaquil deberá elaborar un plan de formación, capacitación Epistemológica, Holística, Axiológica, Humanista permanente, como política Institucional, para que el gremio de Docente, fortalezca y actualice sus conocimientos de manera continua en el desarrollo de estudios superiores de posgrado, deberán ser gratuitos cien por ciento, en virtud que sus remuneraciones son muy paupérrimas. Con la actualización académica de sus Profesores, la Facultad de Ingeniería Industrial de la Universidad de Guayaquil obtendrá como resultado la Optimización del aprendizaje humanista de todos sus estudiantes, consiguiendo como objetivo o producto final la obtención de brillante Ingenieros Industriales con humanismo para el desarrollo productivo del Ecuador.

\section{Agradecimiento}

A Dios nuestro creador por otorgarnos la vida, salud, fuerza y el valor que nos brinda cada día de nuestra existencia para continuar con el trabajo y la lucha permanente hasta conseguir nuestro objetivo en calidad de Docentes de la Facultad Ingeniería Industrial Universidad de Guayaquil.

\section{Bibliografía}

Alvarez, B. (2010). Educacion agenda siglo XXI hacia un desarrollo Humano. Santafé de Bogotá, Colombia: PNUD. Obtenido de https://searchworks. stanford.edu/view/3979381

Figueroa Rubalcava, A., Gilio Medina, M., \& Gutiérrez Marfileño, V. (2008). La funcion del docente en la universidad. Revista electrónica de investigación educativa, 10(Esp), 1-14. Obtenido de http:// www.scielo.org.mx/scielo.php?script=sci_arttex$\mathrm{t} \& \mathrm{pid}=$ S1607-40412008000300008

Madrid Izquierdo, J. (2005). La formación y la evaluación docente del profesorado universitario ante el espacio europeo de educación superior. Educatio Siglo XXI, 23(1), 49-68. Obtenido de https:// revistas.um.es/educatio/article/view/117

Marquez, G. G. (2008). La educacion sera organo maestro desde la cuna hasta la tumba.

Muñoz, A. X. (2011). Regimen Laboral en el Sector Publico. Esmeraldas, Ecuador: Unirsidad Tecnica Particular de Loja.

Osa, G. C. (2008). tendencias educativas para el siglo XXI. Universidad Javeriana.

Perrenoud, P. (2001). La formación de los docentes en el siglo XXI. Revista de Tecnología Educativa, 14(3), 503-523. Obtenido de http://academicos. iems.edu. $\mathrm{m} \times /$ cired/docs/tg/macroacademiaquimica/La\%20formacion\%20de\%20los\%20docentes\%20en\%20el\%20siglo\%20XXI_Perrenoud.pdf

Vasquez, V. (2014). Introduccion Rol Docente. Universidad de Guayaquil.

Vasquez, V. (2014). Resumen Rol del Docente del siglo XXI. Universidad de Guayaquil. 


\section{CITAR ESTE ARTICULO:}

Vásquez Aguilar, D., Soto Chávez, L., Sanchez Granja, A., \& Chang Camacho, L. (2020). Aprendizaje humanista y su aplicabilidad en aula de clases. RECIMUNDO, 4(1(Esp)), 164-172. doi:10.26820/recimundo/4.(1).esp.marzo.2020.164-172

\section{(c) (1) $\circledast($ \\ BY NC SA}

RECONOCIMENTO-NOCOMERCIAL-COMPARTIRIGUAL CC BY-NC-SA
ESTALCENCIA PERMITEA ATROS ENTREMEZCLAR, AUSTARY CONSTRUIR A PARTIR DE SU OBRA CON FINES NO COMERCIALES, SIEMPRE Y CUANDO LE RECONOZCAN LA AUTORÍA Y SUS NUEVAS CREACIONES ESTÉN BAJO UNA LICENCIA CON LOS MISMOS TÉRMINOS. 